Check for updates

Cite this: RSC Adv., 2019, 9, 13145

Received 16th February 2019

Accepted 15th April 2019

DOI: 10.1039/c9ra01206k

rsc.li/rsc-advances

\section{Green electrochemical strategy for one-step synthesis of new catechol derivatives $\dagger$}

\author{
Arafat Toghan, (D) *ab Ahmed M. Abo-Bakr, (D) ${ }^{a}$ Hesham M. Rageha \\ and Mohamed Abd-Elsabour ${ }^{a}$
}

In this paper, we present promising results in the green electrochemical oxidation of catechol in the presence of three different thiol nucleophiles at the surface of a glassy carbon electrode in an aqueous solution using cyclic voltammetry (CV). The outcome indicated the synthesis of some new heterocyclic compounds functionalized with phenolic, triazole, triazine and pyrimidine groups. The effects of repetitive cycling, nucleophile concentrations and sweep rates were explored to get more information about the systems. The voltammetric data showed that the electro-generated o-benzoquinone is a quite reactive intermediate, which in aqueous solutions can quickly participate in a Michael-addition reaction with any one of the nucleophiles to form the corresponding products. The structures of all newly electro-synthesized compounds were confirmed by elemental analyses and FT-IR, ${ }^{1} \mathrm{H} N M R,{ }^{13} \mathrm{C}-\mathrm{NMR}$ and MS spectra. The one-pot synthesis strategy led to new organics with high purities and good yields under green conditions without harmful reagents.

\section{Introduction}

The construction of heterocyclic compounds and their derivatives is highly significant in organic synthesis. ${ }^{1}$ Considering the fact that the majority of pharmaceutical and agrochemical products contain at least one heterocyclic unit, we can most certainly see the importance of heterocyclic compounds. Additionally, many natural products are based on heterocyclic units as well as some of the biologically active compounds such as antibiotics, vitamins, and hormones..$^{2,3}$ Meanwhile, catechol has many similar applications; ${ }^{4,5}$ therefore, many researchers are looking forward to synthesize new catechol derivatives. The combination of catechol and heterocyclic rings, expectedly, will produce new organic products that might be more effective and applicable.

However, the synthesis of these very important organic compounds by classical methods still involves the use of not only strong acids or bases, but also toxic reagents and expensive catalysts. Furthermore, in the conventional methods, the experiments are often carried out at elevated temperatures and require a lot of time (i.e., they are time-consuming). Therefore, many researchers are looking at alternative approaches, i.e., the green chemistry technology for organic synthesis. Recently, electrochemical methods have attracted a lot of attention as

${ }^{a}$ Chemistry Department, Faculty of Science, South Valley University, Qena 83523, Egypt.E-mail: arafat.toghan@yahoo.com

${ }^{b}$ Chemistry Department, College of Science, Imam Mohammad Ibn Saud Islamic University (IMSIU), Riyadh 11623, Kingdom of Saudi Arabia

$\dagger$ Electronic supplementary information (ESI) available. See DOI: $10.1039 / \mathrm{c} 9 \mathrm{ra} 01206 \mathrm{k}$ promising and versatile methods for the preparation of these organic compounds. ${ }^{6,7}$ The reason for this is that the organic derivative synthesis can be achieved without the use of toxic and hazardous reagents. Also, it is a cost-effective method with highquality products, high functional group tolerance, scalability, sustainability, low processing temperatures (mild conditions), and low energy solution process, which can be carried out under environmentally friendly conditions; therefore, it can be used as an alternative method. ${ }^{8,9}$

Due to aromatic $\mathrm{OH}$ groups, phenolic compounds act as antioxidants via an electron transfer process. ${ }^{10}$ Antioxidant activity seems to be related to a range of factors, and the most important are the molecular structure, number of $\mathrm{OH}$ groups, bioavailability, the oxidation potential of the antioxidant and the stability and reactivity of the subsequent phenoxyl radical. A large number of electrochemical methods have been applied successfully for evaluating the antioxidant capabilities of these compounds. ${ }^{11-13}$ So far, many research groups have pursued a possible correlation between the oxidation potentials and antioxidant activity of phenolic acids. ${ }^{14-16}$ Accordingly, it was reported that catechol is more easily electrochemically oxidized into the corresponding $o$-quinone due to its antioxidant activity, low oxidation potential and its ability to form a stable quinoid unit. ${ }^{17,18}$

Indeed, so far, rather detailed studies have been performed on the electrochemical oxidation of catechol in the absence and presence of various nucleophiles; ${ }^{19-21}$ however, in this paper, for the first time, we used new and different types of nucleophiles (Scheme 1). They consist of activated five- and six-membered rings of thiols containing nitrogen; these include triazole, 


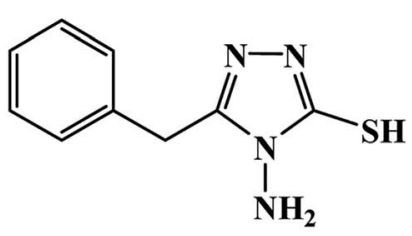

3

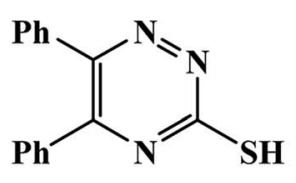

4

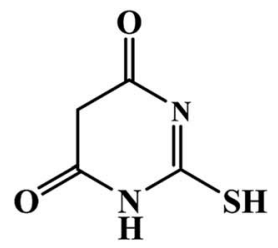

5

Scheme 1 The chemical structures of heterocyclic nucleophiles (3-5).

triazine and pyrimidine rings, which are likely to be useful in the study of the architecture of new drugs. In view of this, the present study led to a straightforward evolution of green electrochemical methods for the synthesis of some new heterocyclic compounds functionalized with phenolic, triazole, triazine, and pyrimidine groups. Specifically, the electrochemical oxidation of catechol was examined in the presence of three different types of heterocyclic nucleophiles in water: (i) 4-amino-5-benzyl$4 H$-1,2,4-triazole-3-thiol (3), ${ }^{22}$ (ii) 5,6-diphenyl-1,2,4-triazine-3thiol (4), ${ }^{23}$ and (iii) 6-mercaptopyrimidine-2,4-(1H,3H)-dione (5).$^{24}$ Interestingly, based on our results, the presented one-pot process (i.e., without the isolation step of intermediates) led directly to the final product $\mathbf{6}, \mathbf{7}$ or $\mathbf{8}$ with high efficiency under eco-friendly conditions, which can be used in a wide range of applications.

\section{Experimental section}

\subsection{Experimental set-up}

The details of the electrochemical experimental conditions have been already described in a previous work. ${ }^{25}$ Briefly, a threeelectrode setup was used: a glassy carbon electrode (GCE, $1 \mathrm{~mm}$ diameter, ALS Co., Ltd) served as the working electrode (WE), saturated $\mathrm{Ag} / \mathrm{AgCl}$ was used as the reference electrode (RE), and a Pt wire (0.6 mm thick) was used as the counter electrode (CE). The RE and CE were obtained from AMETEK Scientific Instruments. Prior to the experiment, the GCE surface was hand polished successfully with a micro-sized abrasive paper and dry alumina powders from Sigma-Aldrich; then, it was immersed in a mixed solution of nitric acid and acetone $(1: 1)$ for $5-10$ minutes. Afterwards, the GCE was immersed in acetone and finally washed by double distilled water to a mirror finish and therefore, it became more active again. The cleaning check of the glassy carbon electrode was evaluated by looking at the difference in the redox peak potential in case of $1 \mathrm{M} \mathrm{H}_{2} \mathrm{SO}_{4}$, where no redox peak is an indication of a clean and active GCE surface.

The electrochemical experiments were performed in EG\&G Princeton applied research potentiostat/galvanostat model 263 controlled by a PC using 352 corrosion software. ${ }^{26}$ Cyclic voltammetry (CV) was used to explore the electrochemical reactions of catechol in the absence and presence of nucleophiles. Before each run, the cell was cleaned with a sulphuric acid solution and then washed with double distilled water. CV tests were conducted at scanning rates of $100 \mathrm{mV} \mathrm{s}^{-1}$ between -1.0 and $+1.0 \mathrm{~V}$ versus the $\mathrm{Ag} / \mathrm{AgCl}$ reference electrode at room temperature.

\subsection{Materials}

All the electrochemical experiments were carried out at room temperature using $1.0 \mathrm{mM}$ catechol (Aldrich Chemical Co. Ltd, 99.8\% purity), $1.0 \mathrm{mM}$ sodium salt of the nucleophilic reagents (3, 4, and 5) and 0.1 $\mathrm{M} \mathrm{Na}_{2} \mathrm{HPO}_{4}$ (El Nasr Pharm. Chem. Co (ADWIC), 98\% purity) as a supporting electrolyte. This concentration was selected after several attempts at different concentrations. All solutions were freshly prepared from analytical grade chemicals without any further purification and dissolved in an appropriate volume of deionized water. Solution $\mathrm{pH}$ was adjusted at a value of $\approx 8$ before the electrolysis experiments by adding few drops of $0.1 \mathrm{M}$ sodium hydroxide (El Nasr Pharm. Chem. Co (ADWIC), 96\% purity) when necessary.

\subsection{General procedures for electro-organic synthesis of catechol derivatives $(6,7$, and 8$)$}

The electrolysis process was carried out in a glass undivided electrolytic cell $\left(\approx 25 \mathrm{~cm}^{3}\right)$ containing $1.0 \mathrm{mM}$ catechol and $1.0 \mathrm{mM}$ sodium salt of the nucleophilic reagent 3,4 , or 5 in aqueous solution of $0.1 \mathrm{M} \mathrm{Na}_{2} \mathrm{HPO}_{4}$ as the supporting electrolyte. The cell was equipped with a Pt-rod anode, Pt-wire cathode, and $\mathrm{Ag} / \mathrm{AgCl}$ as a reference electrode. The reaction mixture was electrolyzed at a constant positive potential of $1.0 \mathrm{~V} v s . \mathrm{Ag} / \mathrm{AgCl}$ for some hours $(\approx 15 \mathrm{~h})$ in air at room temperature. The electrolysis process was interrupted several times to wash the anode for reactivation. At the end of electrolysis, a few drops of acetic acid were added to the solution for acidification and then, the cell was left for a long time until complete dryness of the residue occurred. Afterwards, the residue was heated in $10 \mathrm{~mL}$ of absolute ethanol and then filtered immediately, i.e., when the solution was still hot to remove the inorganic salts. Thereafter, the filtrate was concentrated to half of its volume, placed in the refrigerator overnight and the obtained precipitate was filtered. This product was washed with cold double distilled water and then crystallized from its proper solvent to form 6, 7 or 8 .

\subsection{Spectroscopic characterizations of the electro- synthesized products $(6,7$, and 8$)$}

Briefly, the structures of all electro-synthesized products were confirmed by elemental analysis, FT-IR, ${ }^{1} \mathrm{H}$ NMR, ${ }^{13} \mathrm{C}-\mathrm{NMR}$ and MS; please see ESI. $\dagger$ 


\section{Results and discussion}

Fig. 1 shows the effect of repetitive CVs during the electrochemical oxidation of $0.1 \mathrm{mM}$ catechol at the surface of GCE in an aqueous solution containing $0.1 \mathrm{M} \mathrm{Na}_{2} \mathrm{HPO}_{4}(\mathrm{pH}=8)$ as the supporting electrolyte at room temperature. In agreement with previous studies, ${ }^{27-29} \mathrm{CV}$ of this system shows an anodic peak $\left(\mathrm{P}_{\mathrm{A}}\right)$ and its corresponding cathodic peak $\left(\mathrm{P}_{\mathrm{C}}\right)$, which is due to the transformation of catechol (1) to $o$-benzoquinone (2) and vice versa via a quasi-reversible two-electron process (please see step 1 in Scheme 2). Surprisingly, no losses in the height of the redox peaks with the impossibility of subsequent scans were observed under repetitive cycling on a solution containing only compound 1, as depicted in Fig. 1. These results suggest (i) the stability of the electrode oxidation reaction product, i.e., electrogenerated $o$-benzoquinone, (ii) no occurrence of any side chemical reactions, and also (iii) no inhibition of the electrode surface under the applied experimental conditions. Additionally, the peak current ratios $\left(\mathrm{PI}_{\mathrm{C}} / \mathrm{PI}_{\mathrm{A}}\right)$ are somehow close to unity, which can also be considered as an evidence for the stability of the produced $o$-benzoquinone at the working electrode surface. ${ }^{25,30}$ However, this behavior deviates upon variation in $\mathrm{pH}$ values (not shown), which might be attributed to any other chemical reactions of the electrochemically produced $o$ benzoquinone such as dimerization, ${ }^{31,32}$ hydroxylation ${ }^{33,34}$ or even oxidative ring cleavage. ${ }^{35,36}$ In addition, the deviation of the peak current ratio from ideality is dependent not only on the $\mathrm{pH}$ value but also on the concentrations of catechol. The cathodic peak current decreases with the increase in catechol concentrations at $\mathrm{pH}=8.5 .^{27}$

The question addressed here is how catechol will electrochemically behave after adding any of the above-mentioned nucleophilic reagents $(3,4$ or 5$)$ under the same experimental conditions? The answer is provided by the cyclic voltammograms presented in Fig. 2. It displays the CVs obtained for $1 \mathrm{mM}$

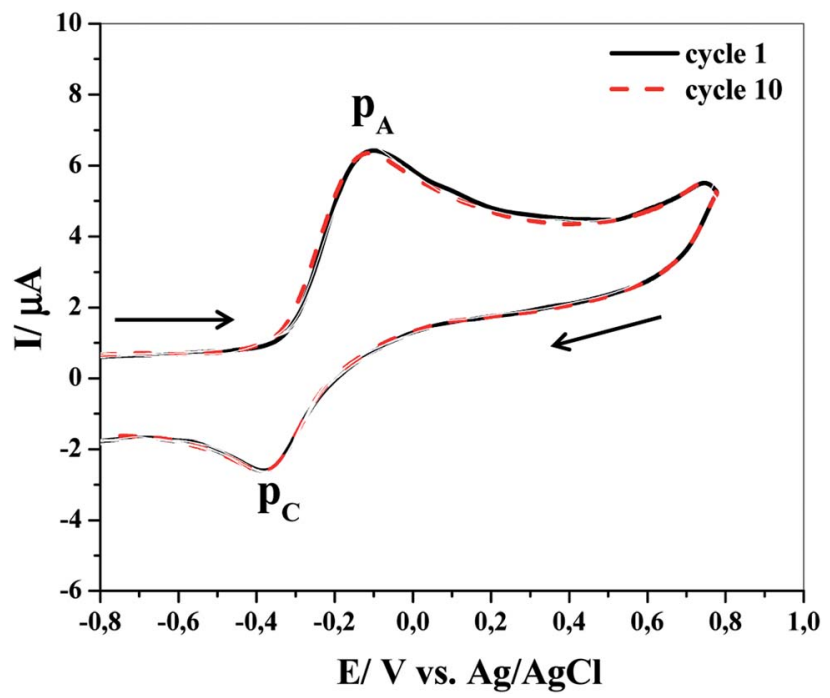

Fig. 1 Effect of repetitive potential cycling in a solution containing $1 \mathrm{mM}$ catechol (1) and supporting electrolyte of $0.1 \mathrm{M} \mathrm{Na}_{2} \mathrm{HPO}_{4}(\mathrm{pH} \approx$ 8 ) at the glassy carbon electrode surface. Scan rate $=100 \mathrm{mV} \mathrm{s}^{-1}$ and temperature $=25^{\circ} \mathrm{C}$. (i) Electrochemical step: electro-oxidation of 1 to 2.

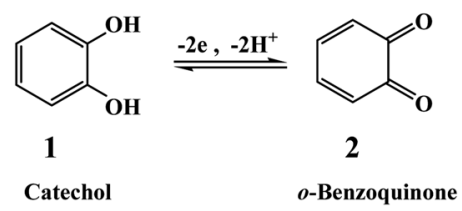

(ii) Chemical step: attacking of 3,4 and 5 to 2 and formation 6,7 and 8, respectively.

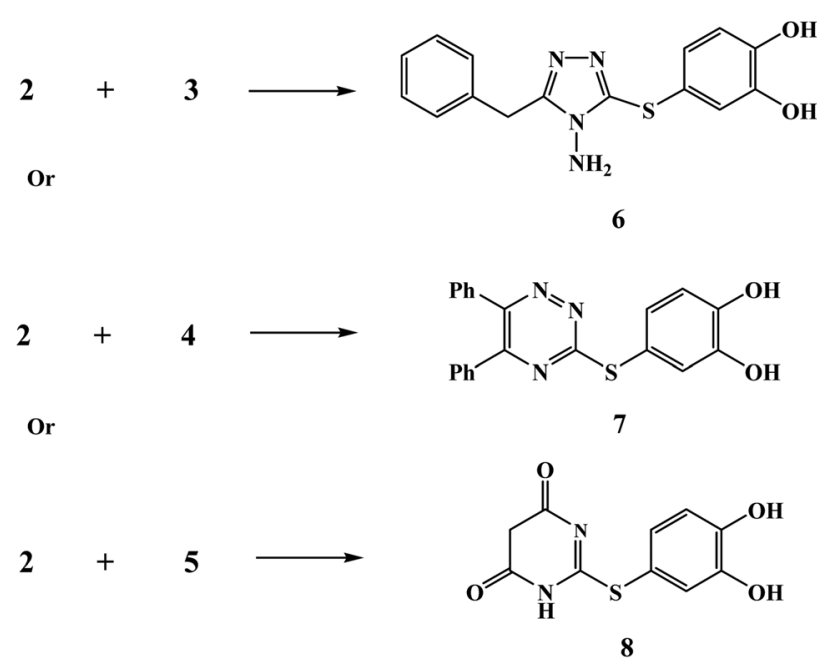

Scheme 2 The electro-transformation of catechol to 0 -benzoquinone and its chemical reactions with 3, 4 or 5 reagents to form 6, 7 or 8 adducts, respectively.

catechol in aqueous solutions $(\mathrm{pH} \approx 8)$ with a scan rate of $100 \mathrm{mV} \mathrm{s}^{-1}$ in the absence and presence of $1 \mathrm{mM}$ of three different nucleophiles 3,4 or 5 . It can be seen from this figure that no redox peaks are observed for pure nucleophiles (i.e., in the absence of catechol), indicating that the nucleophiles 3-5 used here are electrochemically inactive in the defined potential window. Interestingly, the addition of the nucleophiles to catechol exhibited remarkable decrease in the anodic peak current in the forward sweep and complete disappearance of the cathodic peak in the reverse scan under the experimental conditions. This denotes that the electro-oxidation process of catechol is followed by a chemical reaction that rapidly removes the electrogenerated $o$-benzoquinone from the electrode surface. This can only occur if the combination between 2 and nucleophilic reagents $\mathbf{3 , 4}$ or $\mathbf{5}$ is much easier and faster than those in the case of the reduction of compound 2 to 1 . In another way, owing to the nucleophilic attack of 3,4 or 5 on the electrochemically produced compound 2 (o-benzoquinone), the concentration of 2 in the reaction layer will reduce; consequently, the cathodic peak $\mathrm{C}_{\mathrm{A}}$ will decrease or even completely vanish. These results are also supported by the voltammetric data shown in Fig. 3, 4 and 5. In short, according to our results, it seems that the electrogenerated intermediate $o$-benzoquinone is quite reactive and therefore can easily and quickly chemically react with 3, 4 or 5 to form 6, 7 or 8 via a Michael addition reaction, respectively (step 2 in Scheme 2).

Notably, all the CVs were measured at the $\mathrm{pH}$ value of $\approx 8$. This $\mathrm{pH}$ was chosen after several trials and tribulations of 

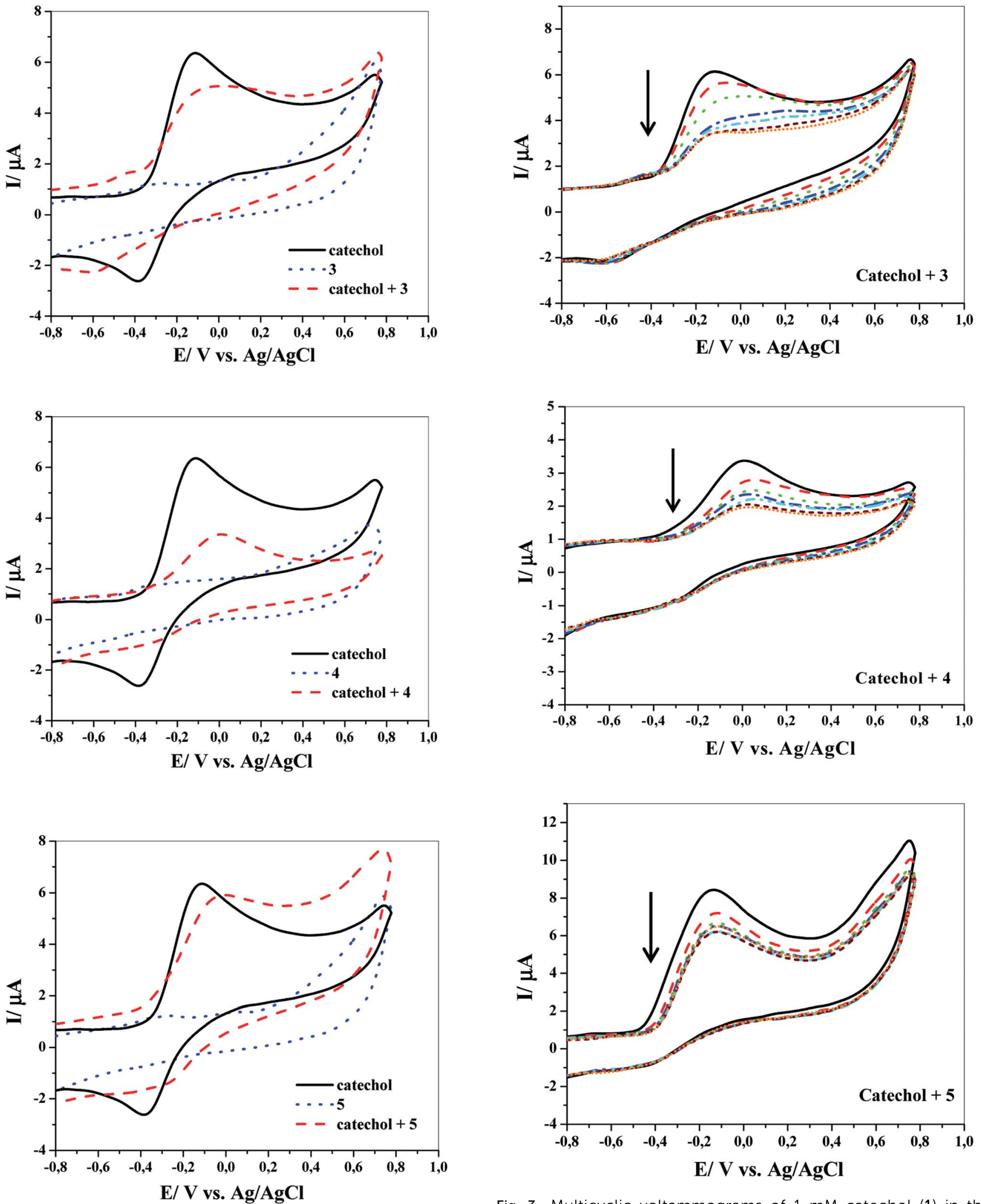

Fig. 2 Cyclic voltammograms recorded at the surface of GC electrode in $0.1 \mathrm{M} \mathrm{Na}_{2} \mathrm{HPO}_{4}(\mathrm{pH} \approx 8)$ solution in the presence and absence of catechol and nucleophile at room temperature and at scan rate $=100 \mathrm{mV} \mathrm{s}^{-1}$. The black solid line represents $1 \mathrm{mM}$ pure catechol (1), the blue dotted line is for $1 \mathrm{mM}$ pure nucleophile $(3,4$ or 5$)$, and the red dashed line denotes a mixture of $1 \mathrm{mM}$ catechol (1) and $1 \mathrm{mM}$ nucleophile $(3,4$ or 5$)$.

Fig. 3 Multicyclic voltammograms of $1 \mathrm{mM}$ catechol (1) in the presence of $1 \mathrm{mM}$ nucleophile (3,4 or 5) in $0.1 \mathrm{M} \mathrm{Na}_{2} \mathrm{HPO}_{4}(\mathrm{pH} \approx$ 8 ) as the supporting electrolyte at room temperature and at a sweep rate of $100 \mathrm{mV} \mathrm{s}^{-1}$. The sweep direction was from up to down. 
different $\mathrm{pH}$ values (data not shown). In fact, this was not surprising because if the $\mathrm{pH}$ values were less than 8 , the thiols would be increasingly protonated and therefore, the soluble sodium salt of the nucleophiles would be converted into insoluble compounds in aqueous solutions. It turned out to be the point of diminishing the nucleophilicity character of the thiol moiety, thus slowing nucleophilic substitution reactions. One can ask a question here: Why they used the sodium salt of the nucleophiles? The answer is not only because the sodium salt of the nucleophiles is soluble in water, but also to keep the sulfur atoms of thiol groups $\left(\mathrm{S}^{-} \mathrm{Na}^{+}\right)$in an ionic form. Consequently, nucleophiles attack catechol via thiol groups only and not through nitrogen, thus exhibiting regioselectivity. Moreover, a $\mathrm{pH}$ of 8 is in quite agreement with recent theoretical/experimental studies, where it was concluded that the hydroxyl group dissociates to its anionic form upon increasing the $\mathrm{pH}$ values..$^{28,29}$ In another way, the proton of $\mathrm{OH}$ group does not participate in the electro-oxidation process; hence, the peak potential is independent of the hydroxyl proton.

The electro-transformation of catechol to the reactive $o$-benzoquinone and its chemical reactions with the nucleophilic reagents 3,4 or 5 to give 6,7 or 8 adducts, respectively, may proceed according to the proposed mechanism shown in Scheme 3. The simultaneous nucleophilic attack of the nucleophiles at the active site (C4) of the oxidized form of catechol (o-benzoquinone) gave an unstable intermediate, which on reduction followed by acidification afforded the mentioned products.
Fig. 3 shows the effect of repetitive CV during the electrochemical oxidation of compound $\mathbf{1}$ in the presence of compound 3, 4 or 5 at GCE in an aqueous medium containing $0.1 \mathrm{M} \mathrm{Na}_{2} \mathrm{HPO}_{4}(\mathrm{pH}=8)$ as the supporting electrolyte at a scan rate of $100 \mathrm{mV} \mathrm{s}^{-1}$. As the number of cycles increase, the anodic peak currents sharply reduce. There are two possible reasons for this to happen: one reason is the contamination or inhibition of the GC electrode surface after the formation of 6,7 or 8 on the reaction layer. An alternative explanation is that the chemical reaction between the electrochemically produced $o$-benzoquinone and nucleophiles leads to decrease in the concentration of catechol. A number of observations strongly support the latter possibility. First, the high electrochemical activity of the electrode depends strongly on the reaction conditions, as demonstrated in Fig. 4; it is sharply reduced after increasing the nucleophile concentration. Second, as shown in Fig. 1, no losses in the electrode activity under repetitive cycling of catechol in the absence of nucleophiles are detected.

More detailed evidence for the occurrence of a chemical reaction between the electrochemically generated $o$-benzoquinone and nucleophiles was obtained by $\mathrm{CV}$ at various scan rates. Fig. 5 shows the cyclic voltammograms obtained for $1 \mathrm{mM}$ catechol in $0.1 \mathrm{M} \mathrm{Na}_{2} \mathrm{HPO}_{4}(\mathrm{pH}=8)$ in the presence of 3,4 or 5 at different scan rates $\left(10-500 \mathrm{mV} \mathrm{s}^{-1}\right)$. Two considerable changes are observed by increasing the scan rate of the experiment. The first is the continued growth of the cathodic peak current while reducing the time window of the experiment

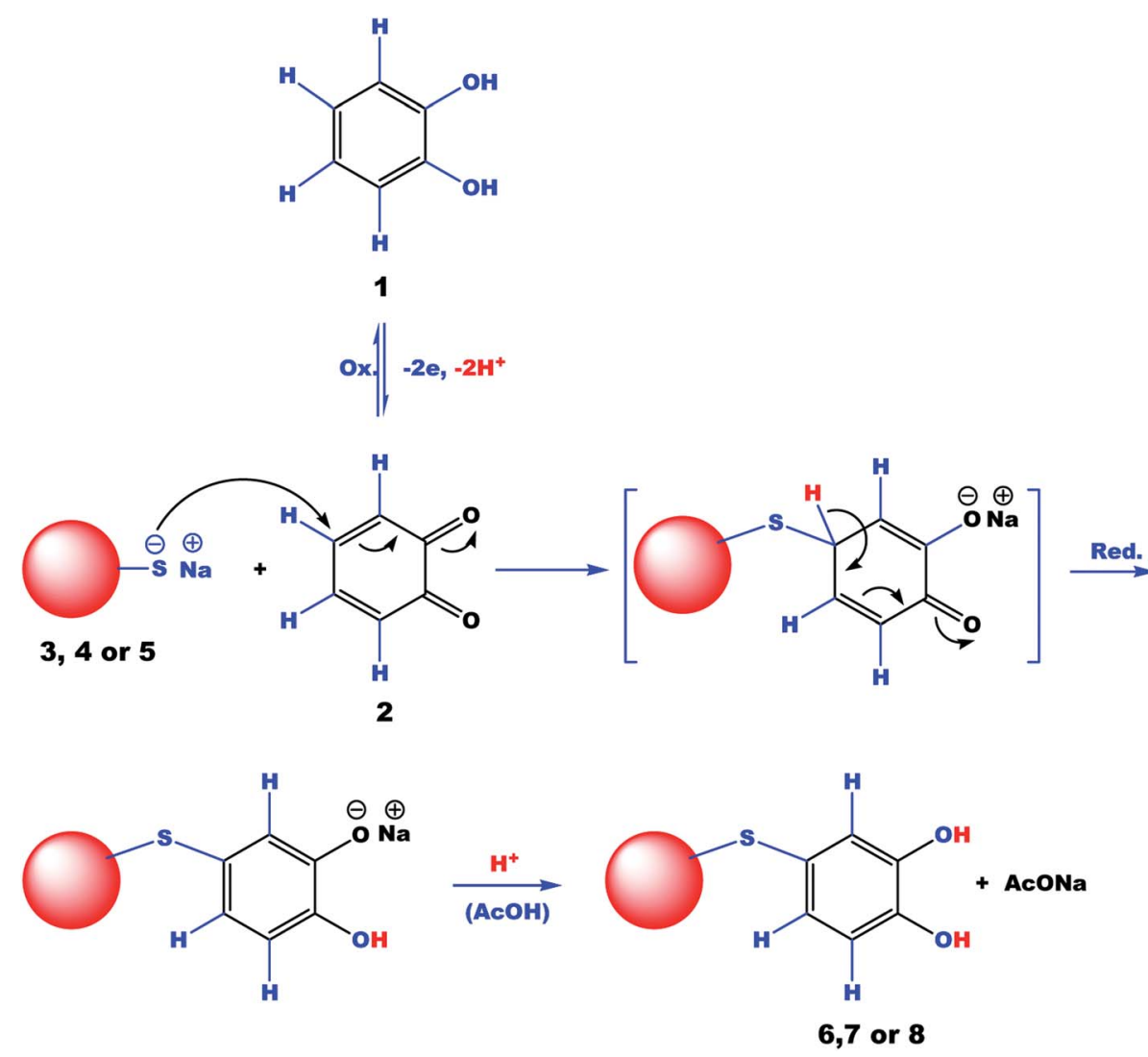

Scheme 3 Proposed mechanism for the electrochemical oxidation of catechol in the presence of three different heterocyclic nucleophiles (3, 4 or 5): one-pot reactions. 

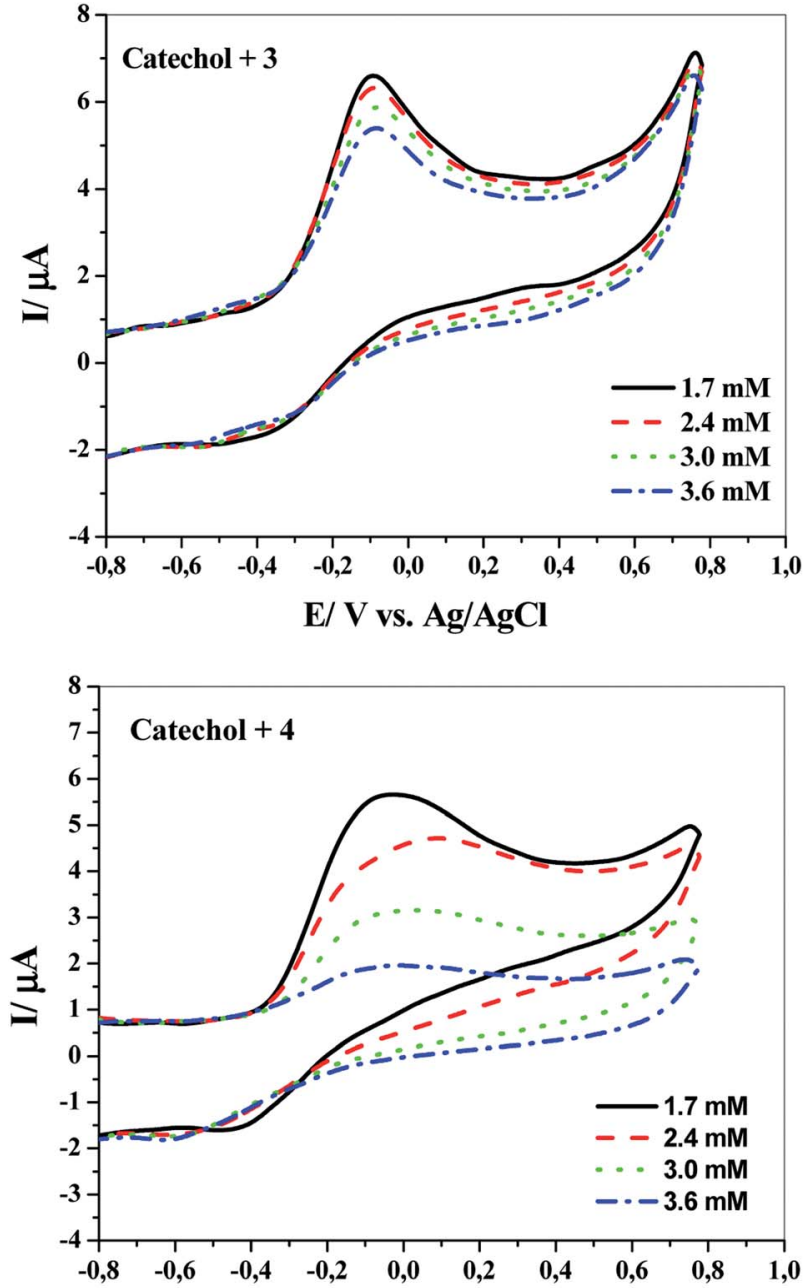

E/ V vs. Ag/AgCl

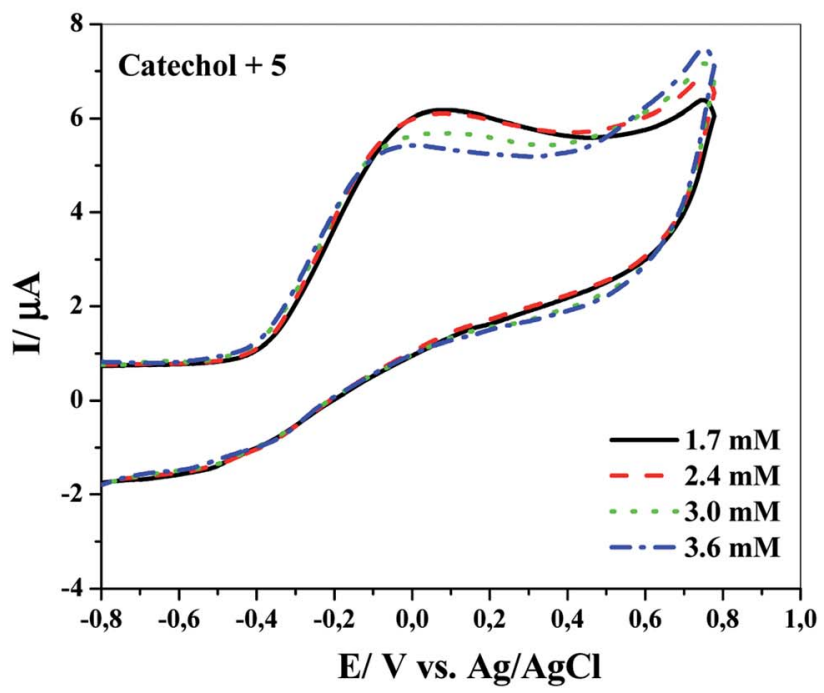

Fig. $4 \mathrm{CV}$ curves show the effect of nucleophilic reagent $(3,4$ or 5$)$ concentrations in the presence of $1 \mathrm{mM}$ catechol (1) and $0.1 \mathrm{M}$ $\mathrm{Na}_{2} \mathrm{HPO}_{4}(\mathrm{pH} \approx 8)$ solution at room temperature and scan rate of $100 \mathrm{mV} \mathrm{s}^{-1}$
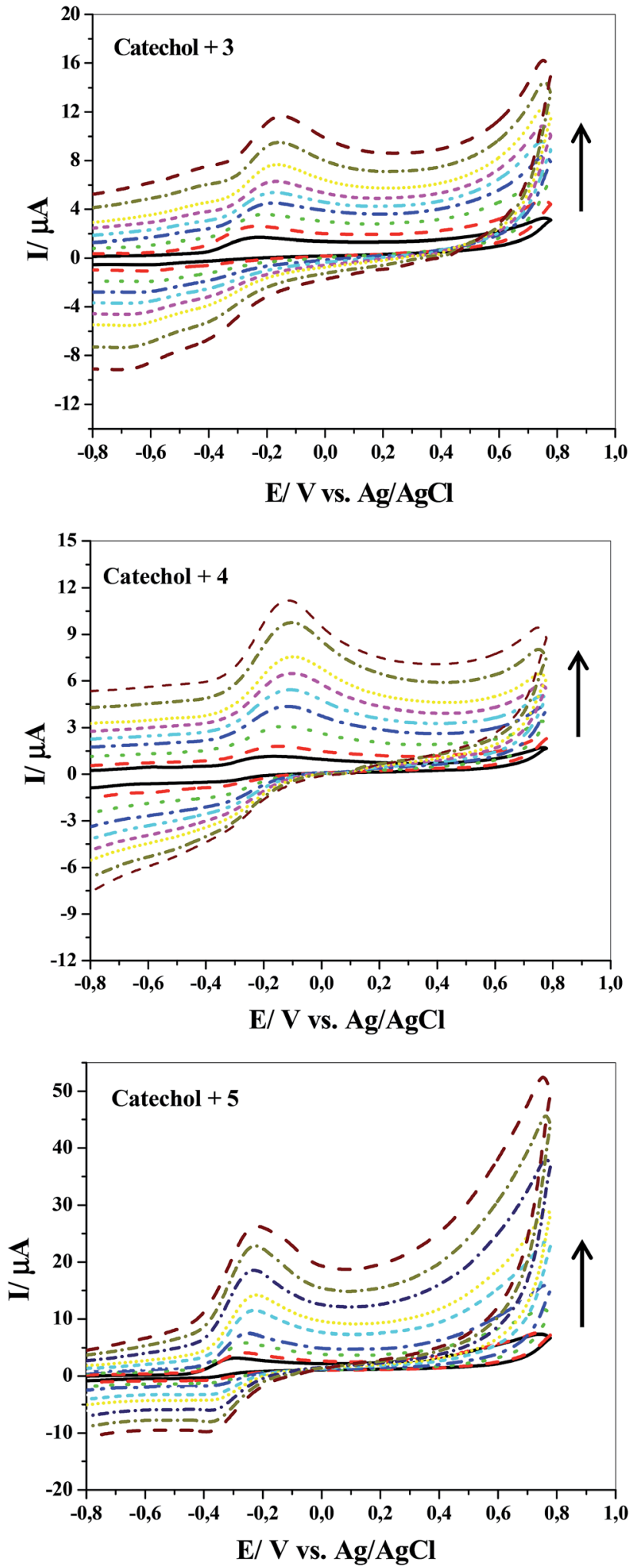

Fig. 5 Typical cyclic voltammograms of $1 \mathrm{mM}$ catechol (1) in the presence of $1 \mathrm{mM}$ nucleophile $(3,4$ or 5$)$ at the GC electrode surface under various scan rates (from down to up) 10, 50, 100, 150, 200, 250, 300,400 and $500 \mathrm{mV} \mathrm{s}^{-1}$. 
(increasing scan rate). Second, the ratios of the cathodic peak to the anodic peak currents are directly proportional to the sweep rates. This result is observed because the extent and progress of the chemical reaction between the electro-generated $o$-benzoquinone and nucleophiles are suppressed at high scan rates; consequently, the amount of product 6,7 or 8 at the surface decreases. In connection with the CV curves shown in Fig. 4 , it is clear that the chemical reaction becomes more favorable at higher concentrations of any of the nucleophilic reagents.

What has not been explained so far is why the potentials of the anodic peaks $\left(P_{\mathrm{A}}\right)$ shift to more positive values and the cathodic peaks $\left(P_{\mathrm{C}}\right)$ to more negative values in the presence of any of the nucleophilic reagents 3,4 or 5 . This is probably due to the accumulation of a thin film of the product 6,7 or 8 at the surface of the WE electrode and therefore, the WE electrode performance is inhibited to a certain extent. ${ }^{37-40}$ All the voltammetric data demonstrated that there were no new redox peaks (i.e., in addition to the original peaks of pure catechol) after adding a nucleophile to catechol under applied experimental conditions. On the one hand, the absence of new redox peaks suggests that no stable intermediates were formed during the electrochemical oxidation of compound 1 in the presence of compound 3. On the other hand, it indicated that the electro-oxidation of the formed compound 6, 7 or 8 was considerably difficult when compared to the electro-oxidation of the parent molecule (compound 1 ) under applied experimental conditions. This could be due to the entrance of an electron-withdrawing group in the catechol ring and/or the insolubility of the formed product in the experimental reaction medium. All these results support the proposed mechanism presented in Scheme 3, which explains how the thiol (-SH group) in the nucleophile $3, \mathbf{4}$ or $\mathbf{5}$ (i.e., as monodentate) attacks catechol over the surface of GCE in consecutive steps; the reaction ends by the formation of the stable product 6,7 , or $\mathbf{8}$ via a Michael addition reaction.

\section{Conclusions}

In this work, the electrochemical oxidation of catechol in the presence of three different heterocyclic nucleophiles (3-5) in water was studied. A solution with a pH value of 8 was selected as a suitable medium for this study. This could be ascribed to the fact that the electro-oxidation of catechol in the presence of thiol nucleophiles was facilitated in weak basic media and hence, the rate of electron transfer was faster. The diagnostic criteria of CVs included vanishing of the cathodic peak in the reverse scan of compound $\mathbf{1}$ in the presence of $\mathbf{3}$ and its reappearance on increasing the sweep rate. The outcome indicated the occurrence of a coupling reaction between the electrogenerated reactive $o$-benzoquinone and compounds $3 \mathbf{- 5}$ as monodentate nucleophiles, leading to the formation of three new catechol derivatives 6-8 with high purities, stabilities and yields via Michael addition one-pot reaction (Schemes 2 and 3) under environmentally friendly conditions.

\section{Conflicts of interest}

There are no conflicts of interest to declare.

\section{References}

1 M. J. Hülsey, H. Yang and N. Yan, ACS Sustainable Chem. Eng., 2018, 6, 5694.

2 C. T. Vo and J. W. Bode, J. Org. Chem., 2014, 79, 2809.

3 S. Bräse, ChemMedChem, 2015, 11, 1107.

4 N. Schweigert, A. J. B. Zehnder and R. I. L. Eggen, Microbiol., 2001, 3, 81.

5 R. H. Blum and S. K. Carter, Ann. Intern. Med., 1974, 80, 249.

6 S. Tang, D. Wang, Y. Liu, L. Zeng and A. Lei, Nat. Commun., 2018, 9, 798.

7 C. Li, K. Yu, H. Nakamura, J. C. Vantourout, Z. Liu, Q. Hou, D. Bao, J. T. Starr, J. Chen, M. Yan and P. S. Bara, Angew. Chem., Int. Ed., 2017, 56, 13088.

8 M. Yan, Y. Kawamata and P. S. Baran, Angew. Chem., Int. Ed., 2018, 57, 4149.

9 A. Li, W. Duan, J. Liu, K. Zhuo, Y. Chen and J. Wang, Sci. Rep., 2018, 8, 13141.

10 C. G. M. Heijnen, G. R. M. M. Haenen, J. A. J. M. Vekemans and A. Bast, Environ. Toxicol. Pharmacol., 2001, 10, 199.

11 P. Janeiro and A. M. O. Brett, Anal. Chim. Acta, 2004, 518, 109-115.

12 H. Hotta, S. Nagano, M. Ueda, Y. Tsujino, J. Koyama and T. Osakai, Biochim. Biophys. Acta, 2002, 1572, 123.

13 Z. Cheng, J. Ren, Y. Li, W. Chang and Z. Chen, Redox Rep., 2002, 7(6), 395-402.

14 S. A. B. E. Van Acker, D. V. D. Berg, M. N. J. L. Tromp, D. H. Griffioen, W. P. V. Bennekom, W. J. F. V. D. Vijgh and A. Bast, Free Radical Biol. Med., 1996, 20, 331-342.

15 R. Toniolo, F. D. Narda, G. Bontempelli and F. Ursini, Bioelectrochemistry, 2000, 51, 193-200.

16 P. A. Kilmartin, H. L. Zou and A. L. Waterhouse, J. Agric. Food Chem., 2001, 49, 1957-1962.

17 R. H. Bisby, R. Brooke and S. Navaratnam, Food Chem., 2008, 108, 1002-1007.

18 M. A. Motin, M. A. Uddin, P. K. Dhar, M. A. H. Miaa and M. A. Hashem, Port. Electrochim. Acta, 2017, 35(2), 103-116.

19 D. Nematollahi, A. A. Taherpour, S. Jameh-Bozorghi, A. Mansouri and B. Dadpou, Int. J. Electrochem. Sci., 2010, $5,867$.

20 A. R. Fakhari, K. Hasheminasab, H. Ahmar and A. Alizadeh, Synthesis, 2008, 24, 3963.

21 D. Nematollahi, L. Mohammadi-Behzad and S. S. H Davarani, Electroanalysis, 2009, 21, 1099.

22 A. Cansız, M. Koparır and A. Demirdağ, Molecules, 2004, 9, 204.

23 H. Irannejad, N. Naderi, S. Emam, R. Q. Ghadikolaei, A. Foroumadi, T. T. Zafar and A. Mazar-Atabaki, Med. Chem. Res., 2014, 23, 2503.

24 h. Biltz and H. Wittek, Chem. Ber., 1921, 54, 1035.

25 A. Toghan, A. M. Abo-baker, H. M. Rageh, M. M. Abou-Krisha and M. Abd-Elsabour, J. Pharm. Appl. Chem., 2018, 4, 133.

26 M. M. Abou-Krisha, F. H. Assaf and A. A. Toghan, J. Solid State Electrochem., 2007, 11, 244.

27 D. Nematollahi, F. Ghasemi, S. Khazalpour and F. Varmaghani, J. Chem. Sci., 2016, 128(12), 1887. 
28 S. Nassif, D. Bakeer, R. Madwar, W. Khadam and A. Nakhla, J. Electrochem. Sci. Eng., 2019, 9(1), 1.

29 M. A. H Mia, M. A. Motin and E. M. Huque, Port. Electrochim. Acta, 2018, 36(6), 437.

30 S. Shahrokhian and A. Hamzehloei, Electrochem. Commun., 2003, 5, 706.

31 M. D. Ryan, A. Yueh and W. Y. Chen, J. Electrochem. Soc., 1980, 127, 1489.

32 D. Nematollahi, M. Rafiee and A. Samadi-Maybodi, Electrochim. Acta, 2004, 49, 2495.

33 L. Papouchado, G. Petrie, J. H. Sharp, N. Ralph and R. N. Adams, J. Am. Chem. Soc., 1986, 90, 5621.

34 E. T. DNematollahi, J. Org. Chem., 2005, 70, 7769.
35 F. Varmaghani and D. Nematollahi, Electrochim. Acta, 2011, 56, 6089.

36 R. A. Izydore, H. E. Johnson and R. T. Horton, J. Org. Chem., 1985, 50, 4589.

37 D. Nematollahi, A. R. Atlasi-Pak and R. Esmaili, Helv. Chim. Acta, 2012, 95, 1605.

38 M. A. Chamjangali, M. Bakherad and M. Ameri, Monatsh. Chem., 2015, 146, 111.

39 L. Fotouhia, S. Asadia, E. Tammari, M. M. Heravia and D. Nematollahic, J. Iran. Chem. Soc., 2008, 5, 712.

40 T. Kashiwagi, F. Amemiya, T. Fuchigami and M. Atobe, J. Flow Chem., 2012, 3, 17. 